\title{
AUFSÄTZE
}

\section{Der Bundespräsident als „Außerparlamentarische Opposition“? Überlegungen zur Gewaltenteilung und Typologisierung des parlamentarischen Regierungssystems}

\author{
Roland Lhotta*
}

Es ist mittlerweile ein Gemeinplatz, dass das politische System der Bundesrepublik Deutschland eine spezifisch gewaltenteilige Struktur aufweist, die über die Institutionalisierung zahlreicher Vetopunkte ${ }^{1}$ politischen Akteuren die Möglichkeit gibt, bei Entscheidungen den Konsensdruck erheblich zu erhöhen und sich dadurch zu Mitregenten aufzuschwingen $^{2}$. Verschieden konstruierte Indizes ${ }^{3}$ haben zudem immer wieder verdeutlicht, dass die institutionellen Begrenzer der Regierungsmacht in der Bundesrepublik das Regieren zu einem mühsamen Geschäft machen. Die Bundesrepublik Deutschland lässt sich deshalb kaum zufällig in die von Arend Lijphart entwickelte Kategorie der Konsensdemokratien einordnen ${ }^{4}$, wobei auch und gerade das parlamentarische System einer Logik gewaltenteiliger Kooperation unterworfen ist, die es einer Reihe institutionalisierter Vetospieler erlaubt, selbst unter Auspizien klarer parlamentarischer Mehrheiten, deren „Durchregieren“ zu verhindern. Hierfür bietet die aktuelle Große Koalition ein gutes Beispiel: „Theoretisch ist die Große Koalition unstürzbar und übermächtig, praktisch jedoch provoziert sie soviel Gegenkräfte, dass von ihrer Macht nichts bleibt. Eigentlich galt die Befürchtung, mit dem Bündnis der Volksparteien verschwinde die Opposition. Nun zeigt sich das Gegenteil: Die Opposition wird allgegenwärtig, aber das Regieren verschwindet. "5

Dies wäre an sich noch kein wirklich aufregender Befund, würde er doch nur bestätigen, dass die gewaltenteilende Mischverfassung des Grundgesetzes ihre institutionalisierten Konsenszwänge eigentlich immer zur Geltung bringen kann, weil die konstitutionellen Opportunitätsstrukturen, die sie für politische Akteure im parlamentarischen Bundesstaat zur Verfügung stellt, das „Mitregieren“ zum Grundprinzip erheben. Eine neue Qualität gewinnt dieses komplexe Mit- und Gegeneinander indessen - glaubt man landläufigen Ver-

* Unter Mitarbeit von Sabrina Zucca.

1 André Kaiser, Vetopunkte der Demokratie. Eine Kritik neuerer Ansätze der Demokratietypologie und ein Alternativvorschlag, in: ZParl, 29. Jg. (1998), H. 3, S. 525 - 541.

2 Vgl. vor allem Manfred G. Schmidt, Regieren in der Bundesrepublik Deutschland, Opladen 1992, S. 42 ff.; ders., Political Institutions in the Federal Republic of Germany, Oxford 2003; ders., Das politische System der Bundesrepublik Deutschland, München 2005.

3 Vgl. ders., Demokratietheorien, 3. Auflage, Opladen 2000, S. $351 \mathrm{ff}$.

4 Vgl. Arend Lijphart, Patterns of Democracy. Government Forms and Performance in Thirty-Six Countries, New Haven / London 1999, S. 312 ff.

5 Matthias Geis / Bernd Ulrich, Die neue APO macht mobil, in: Die Zeit vom 18. Januar 2007, S. 4. 
lautbarungen aus der jüngsten Vergangenheit - durch einen Bundespräsidenten, der als „erster Opponent der Regierenden“ und Bestandteil einer neuen „Außerparlamentarischen Opposition“ (APO) aus der Mitte der Republik agiere, um sich damit als „ernsthafter politischer Gegenspieler“ der regierenden Mehrheit in Gestalt einer Großen Koalition zu präsentieren ${ }^{6}$. „Zupacken, angreifen, dazwischengrätschen “7 - dies seien die primären Interessen des "großen Vorgesetzten "8 auf seinem Weg zum politischsten Bundespräsidenten aller Zeiten.

\section{Prüfungsrecht und Ausfertigungsverweigerung: sektorale Präsidentialisierung des parlamentarischen Systems?}

Die soeben vorgetragene - etwas reißerische - Diagnose, die sich vor allem an der als Einladung zur Verfassungsbeschwerde verstandenen Formulierung schwerer Bedenken gegen das Luftsicherheitsgesetz ${ }^{9}$ sowie der Weigerung des Bundespräsidenten entzündete, zwei Gesetze der Großen Koalition auszufertigen ${ }^{10}$, hat nicht unerhebliche Implikationen: Sie pflanzt in das ohnehin durch widerstreitende Handlungslogiken im Gefolge der bundesstaatlichen Struktur ${ }^{11}$ geprägte parlamentarische System eine präsidentielle Komponente ein, die das Staatsoberhaupt als nachträglichen und fallabhängigen Vetospieler ${ }^{12}$ etabliert. Damit würde der durch das Attribut einer Kanzlerdemokratie bereits als „semi-parliamentarism“13 apostrophierte deutsche Parlamentarismus zusätzlich modifiziert und rückte ein weiteres Stück vom „pure parliamentarism“ ab. Die Richtung dieser Modifikation klingt in der Analyse semi-präsidentieller Systeme von Matthew Shugart an, wenn er feststellt, dass ein präsidentielles Veto „may result in a transactional situation that would not occur in a parliamentary

6 Ebenda, S. 4.

7 Sebastian Fischer, Zupacken, angreifen, dazwischengrätschen, in: Spiegel Online vom 14. Dezember 2006 (http://www.spiegel.de/politik/deutschland/0,1518,454340,00.html).

8 Robert Leicht, Der große Vorgesetzte, in: Die Zeit vom 20. Dezember 2006, S. 12.

9 Der Bundespräsident hielt die $\$ \$ 13$ und 14 III LuftSiG für „,verfassungsrechtlich höchst bedenklich“ und erklärte, mit offensichtlichem Bedauern, er sei im Gegensatz zum Bundesverfassungsgericht „nicht befugt, ein mir zur Ausfertigung vorgelegtes Gesetz nur teilweise in Kraft zu setzen“. Mit seiner Entscheidung mache er „den Weg frei für eine verfassungsgerichtliche Überprüfung, die jeder Betroffene auch unter Hinweis auf die von mir aufgezeigten Bedenken durch das Bundesverfassungsgericht vornehmen lassen kann" (http://www.bundespraesident.de/Journalistenservice/Pressemitteilungen-, 11107.621599/Bundespraesident-Horst-Koehler.htm?global.printview=2). Die hierauf erfolgte Verfassungsbeschwerde wurde vom BVerfG angenommen und $\$ 14$ III LuftSiG im Urteil des Ersten Senats vom 15. Februar 2006 als gleich mehrfach mit dem Grundgesetz unvereinbar und nichtig deklariert.

10 Es handelte sich um das Gesetz zur Neuregelung des Rechts der Verbraucherinformation sowie um das Gesetz zur Neuregelung der Flugsicherung; vgl. hierzu die Begründungen für die Nichtausfertigung durch den Bundespräsidenten vom 24. Oktober 2006 und 8. Dezember 2006 in den Pressemitteilungen unter www.bundespraesident.de sowie BT-Drs. 16/3866.

11 Gerhard Lehmbruch, Parteienwettbewerb im Bundesstaat. Regelsysteme und Spannungslagen im Institutionengefüge der Bundesrepublik Deutschland, 3., erweiterte Auflage, Wiesbaden 2000.

12 Für diese Kategorisierung Michael Stoiber, Gewaltenteilung, Machtteilung und das VetospielerKonzept, in: ZPol, 17. Jg. (2007), S. 21 - 41, S. 33 f.

13 Giovanni Sartori, Neither Presidentialism nor Parliamentarism, in: Juan Linz / Arturo Valenzuela (Hrsg.), The Failure of Presidential Democracy, Baltimore / London 1994, S. 106 - 118, S. 110. 
system: the inability of a government to pass a law with a plurality while some parties or members either abstain or are absent from the vote "14. Nun kann man natürlich, wie es die deutsche Staatsrechtslehre praktiziert, darauf verweisen, dass es allen Verfassungsorganen obliege, „von vorneherein die verfassungsrechtlichen Erfordernisse für staatliches Handeln zu beachten " 15 und es somit durchaus in der Logik der bundesrepublikanischen Mischverfassung liege, dass der Bundespräsident „,in funktionaler und gewaltenteiliger Verschränkung mit anderen Organen“ an der Ausübung der Staatsgewalt teilnehme ${ }^{16}$ und dabei als „rechtliche Reserve“ einspringen könne, „wenn auf andere Weise ein verfassungswidriges Zusammenwirken der anderen Verfassungsorgane nicht verhindert werden kann"17. Dies gelte erst recht, wenn davon auszugehen sei, dass der Bundespräsident im deutschen System der Gewaltenteilung nicht eindeutig zuzuordnen, vielmehr ,sozusagen komplementär zu allen übrigen Verfassungsorganen angelegt ist, und zwar nicht im Sinne einer Ersatzfunktion, sondern aus einer eigenen Verfassungsposition heraus ergänzend und unterstützend" 18 . Dann stellt sich aber die Frage - wenn auf der anderen Seite der Grundsatz der Gewaltenteilung nach Art. 20 Abs. 2 GG ,gegen materielle Eingriffe des Bundespräsidenten als eines nicht der Legislative angehörenden Verfassungsorgans in die Gesetzgebung “ spricht ${ }^{19}$-, ob Verschränkung und Mischverfassung, speziell fokussiert im Vetopunkt der Ausfertigungsverweigerung, nicht zu einer sektoralen Präsidentialisierung des parlamentarischen Systems führen können, die eine Klassifizierung der Bundesrepublik als parlamentarisches System als zumindest gelegentlich zweifelhaft oder kontingent erscheinen lässt ${ }^{20}$.

Zwar stimmte die Diagnose nach wie vor, dass sich eine Bundestagsmehrheit sowie die von ihr getragene Regierung „einem tiefgestaffelten System von institutionellen Checks and Balances gegenüber“ sehen; dass aber der Bundespräsident als „klassisches Korrektiv des Parlaments" nunmehr durch andere politische Institutionen wie Bundesrat und Bundesverfassungsgericht als Schranken parlamentarischer Mehrheitsherrschaft substituiert sei ${ }^{21}$, mag dann doch ein etwas voreiliger Schluss gewesen sein. Damit bleibt die Frage, ob das Prüfungs- und Ausfertigungsverweigerungsrecht des Bundespräsidenten „in gewisser Weise

14 Matthew S. Shugart, Semi-Presidential Systems: Dual Executive and Mixed Authority Patterns, in: French Politics, 3. Jg. (2005), S. 323 - 351, S. 339.

15 Christoph Degenhart, Staatsrecht I. Staatszielbestimmungen, Staatsorgane, Staatsfunktionen, 13., völlig neu bearbeitete Auflage, Heidelberg 1997, S. 195, Rz. 465.

16 Martin Nettesheim, Amt und Stellung des Bundespräsidenten, in: Josef Isensee / Paul Kirchhof (Hrsg.), Handbuch des Staatsrechts der Bundesrepublik Deutschland, Bd. 3, 3., völlig neubearbeitete und erweiterte Auflage, Heidelberg 2005, S. 1054 f., Rz. 41.

17 Klaus Schlaich, Die Funktionen des Bundespräsidenten im Verfassungsgefüge, in: Josef Isensee I Paul Kirchhof (Hrsg.), Handbuch des Staatsrechts der Bundesrepublik Deutschland, Bd. 2, Heidelberg 1987, S. 559 f., Rz. 41.

18 Jürgen Jekewitz, Der Bundespräsident, vor Art. 54, S. 479, Rz. 18, in: Kommentar zum Grundgesetz für die Bundesrepublik Deutschland (Reihe Alternativkommentare, hrsg. von Rudolf Wassermann), Bd. 2, Neuwied / Darmstadt 1984 (Hervorhebung des Verfassers).

19 Christoph Degenhart, a.a.O., S. 195, Rz. 465.

20 Notabene: Damit ist nicht eine Gefährdung des Grundsatzes der Gewaltenteilung gemeint, der in der Tat „durch Veränderung einzelner seiner Bausteine nicht sogleich ins Wanken geraten“ kann - so zutreffend Andreas von Arnauld, Gewaltenteilung jenseits der Gewaltentrennung. Das gewaltenteilige System in der Verfassungsordnung der Bundesrepublik Deutschland, in: ZParl, 32. Jg. (2001), H. 3, S. $678-698$, S. 686.

21 Wolfgang Rudzio, Das politische System der Bundesrepublik Deutschland, 7., aktualisierte und erweiterte Auflage, Wiesbaden 2006, S. 300. 
systemwidrig“22, das heißt letztlich unvereinbar mit dem parlamentarischen System ist ${ }^{23}$, bestehen - denn immerhin führt die Ausfertigung durch den Bundespräsidenten zur Verkündung und damit zum Wirksamwerden der Gesetze; die Verweigerung der Ausfertigung hingegen lässt ein Gesetz, „obgleich von den gesetzgebenden Körperschaften beschlossen, nicht wirksam werden. Im modernen Rechtsstaat mit seiner umfassenden schriftlichen Fixierung des Rechtes ist die Gesetzgebung, also die Ergänzung und Veränderung des geschriebenen Rechts, die zentrale Staatsfunktion. Die Befugnis, Gesetzgebungsakte zu verhindern, ist daher von erheblichem staatsrechtlichen Gewicht " 24 sowie von Bedeutung für die politikwissenschaftliche Typologisierung und Evaluation politischer Systeme beziehungsweise für die Effekte konstitutionalisierter „institutional choices“.

\section{Der Bundespräsident als institutioneller Vetospieler: eine doppelte Herausforderung für die Typen-und Gewaltenteilungslehre}

Mit dem Präsidenten als Vetospieler ist ein institutionelles Design angesprochen, das aus der Perspektive des neo-institutionalistischen Mainstreams der Politikwissenschaft dazu herausfordert, Schlüsse zu ziehen über „outcomes of institutional choice“25, gleichzeitig aber Probleme bereitet, denn „there is thus not only no definitional role of a ,president ' in a parliamentary system, but not even an agreed starting point for viewing the position " 26 . Dies führte bekanntermaßen schon Anfang der 1980er Jahre zu der durch Maurice Duverger initiierten Auseinandersetzung mit dem Typus des semi-präsidentiellen Systems, wobei das zentrale Problem in der großen Varianz liegt ${ }^{27}$, die die Ausgestaltung des Präsidentenamtes in politischen Systemen erfahren hat. Dies wiederum zog immer feingliedrigere Typologien nach sich ${ }^{28}$, oder aber der Semi-Präsidentialismus als eigenständiger Systemtyp wurde rundweg zurückgewiesen ${ }^{29}$. Ohne hier vertiefend in diese Debatte einsteigen zu können ${ }^{30}$, scheint der Dreh- und Angelpunkt der Auseinandersetzung die Machtausstat-

22 Klaus von Beyme, Das politische System der Bundesrepublik Deutschland. Eine Einführung, 9., neu bearbeitete und aktualisierte Auflage, Opladen / Wiesbaden 1999, S. 332.

23 Vgl. Werner Kaltefleiter, Die Funktionen des Staatsoberhauptes in der parlamentarischen Demokratie, Köln 1970, S. 275.

24 Joachim Mewing, Die Prüfungskompetenz des Bundespräsidenten bei der Gesetzesausfertigung, insbesondere beim teilnichtigen Gesetz, Berlin 1977, S. 22.

25 Robert Elgie, Semi-Presidentialism: Concepts, Consequences and Contesting Explanations, in: Political Studies Review, 2. Jg. (2004), S. 314 - 330, S. 317.

26 Alan Siaroff, Comparative Presidencies: The Inadequacy of the presidential, semi-presidential and parliamentary distinction, in: European Journal of Political Research, 42. Jg. (2003), S. 287 -312 , S. $289 \mathrm{f}$.

27 Matthew S. Shugart, Comparative Executive-Legislative Relations, in: R. A. W. Rhodes / Sarah A. Binder / Bert A. Rockman (Hrsg.), The Oxford Handbook of Political Institutions, Oxford 2006, S. $344-365$, S. 350 .

28 Die bekannteste und für die weitere Diskussion wohl fruchtbarste Typologie wurde vorgelegt von Matthew S. Shugart / John M. Carey, Presidents and Assemblies. Constitutional Design and Electoral Dynamics, Cambridge 1992.

29 Vgl. insbesondere Winfried Steffani, Semi-Präsidentialismus: ein eigener Systemtyp? Zur Unterscheidung von Legislative und Parlament, in: ZParl, 26. Jg. (1995), H. 4, S. 621 - 641.

30 Einen kurzen Überblick geben jeweils Stefan Marschall, Parlamentarismus. Eine Einführung, Baden-Baden 2005, S. 61 ff., sowie Aurel Croissant, Regierungssysteme und Demokratietypen, in: 
tung des Präsidenten zu sein beziehungsweise die Möglichkeit, diese Macht auch gegenüber dem Parlament und der aus dem Parlament hervorgehenden Regierung zur Geltung zu bringen. Dieser Aspekt kommt bereits in einem Definitionsmerkmal bei Duverger zum Tragen, wenn er konstatiert, dass in semi-präsidentiellen Systemen der Präsident über „quite considerable powers“ verfüge ${ }^{31}$. Aber was heißt das eigentlich? Und ab wann beziehungsweise welcher Machtausstattung des Präsidenten wird ein parlamentarisches System in die Richtung eines präsidentiellen oder semi-präsidentiellen verschoben ${ }^{32}$ ? Die Möglichkeit jedenfalls, ein von der Parlamentsmehrheit getragenes Gesetz nicht auszufertigen und damit faktisch über ein (nicht überstimmbares) Veto zu verfügen, scheint eine der Variablen zu sein, die hier eine Rolle spielen ${ }^{33}$ : „Even if the president has no discretion in the forming of cabinets or the right to dissolve parliament, his or her constitutional authority can be regarded as ,quite considerable' in Duverger's sense if cabinet legislation approved in parliament can be blocked by the people's elected agent." 34

Der Umstand, dass im bundesrepublikanischen Fall der Bundespräsident mangels direkter Wahl nicht als „people’s elected agent“ auftreten kann, mag zunächst dafür sprechen, dass damit eines der wichtigsten strukturellen Merkmale präsidentieller und semi-präsidentieller Systeme ${ }^{35}$ nicht gegeben sei und das materielle Prüfungsrecht samt Recht zur Ausfertigungsverweigerung ohnehin nur bei evidenten Verfassungsverstößen zur Geltung komme. Dann - aber auch nur dann - gehe es der Einschätzungsprärogative des bona fide handelnden Parlaments aus Gründen der Wahrung der Verfassungsordnung vor. ${ }^{36}$ Arend Lijphart hat aber zu Recht darauf hingewiesen, dass die duale (konkurrierende) demokratische Legitimation nicht das eigentliche Problem darstellt, denn alle konsensdemokratischen Arrangements, zu denen neben Präsidentialismus etwa auch Föderalismus und Bikameralismus zählten, „may be seen as attempts to prevent a single ,democratic legitimacy', which would necessarily be a single concentration of power “37. Mit der konsensdemokratisch angelegten Wendung gegen eine solche "single concentration of power" ist bereits ein deutliches Echo aus den Federalist Papers vernehmbar, die gerade zu diesem Zweck eine strikte und schematische Gewaltenteilung qua „institutional design” unterlaufen - und zwar mit dem bezeichnenden Argument, ,that a mere demarcation on parchment of the constitutional limits of

Hans-Joachim Lauth (Hrsg.), Vergleichende Regierungslehre. Eine Einführung, Wiesbaden 2002, S. 131 - 155, S. 133 ff.; ausführlicher Robert Elgie, The Politics of Semi-Presidentialism, in: ders. (Hrsg.), Semi-Presidentialism in Europe, Oxford 1999 (Wiederdruck 2004), S. 1 - 21, und ders., Semi-presidentialism: concepts, consequences, and contesting explanations, in: Political Studies Review, 2. Jg. (2004), S. $314-330$.

31 Maurice Duverger, A new Political System Model: Semi-Presidential Government, in: European Journal of Political Research, 8. Jg. (1980), S. 165 - 187, S. 166.

32 Vgl. Alan Siaroff, a.a.O., S. 290.

33 Vgl. Arend Lijphart, Presidentialism and Majoritarian Democracy: Theoretical Observations, in: Juan J. Linz / Arturo Valenzuela (Hrsg.), a.a.O., S. 91 - 105, S. 99 f.

34 Matthew S. Shugart, Semi-Presidential Systems, a.a.O, S. 339.

35 Vgl. etwa Alfred Stepan / Cindy Skach, Constitutional Frameworks and Democratic Consolidation. Parliamentarism versus Presidentialism, in: World Politics, 46. Jg. (1993), S. 1 - 22, S. 4 , sowie Juan J. Linz, Presidential or Parliamentary Democracy: Does it make a Difference, in: ders. I Arturo Valenzuela (Hrsg.), a.a.O., S. 3 - 87, S. 6.

36 Norbert K. Riedel / Axel Schmidt, Die Nichtausfertigung des Gesetzes zur Privatisierung der Flugsicherung durch den Bundespräsidenten, in: DÖV 1991, S. 371 - 375, S. 374.

37 Arend Lijphart, a.a.O. (1994), S. 102. 
the several departments is not a sufficient guard against those encroachments which lead to a tyrannical concentration of all the powers of government in the same hands" 38 . Diese Logik klingt übrigens auch im Vetospieler-Ansatz von George Tsebelis nach, die unter anderem davon ausgeht, dass die Machtteilung in dem Maße zunimmt, wie die Anzahl der Vetospieler steigt. ${ }^{39}$

In dieser Logik ist eine bona fide-Vermutung gegenüber der Legislative beziehungsweise der dort obwaltenden Mehrheiten nicht angezeigt, erst recht bei starken parlamentarischen Mehrheiten. Genau dies kann gegenwärtig im Verhältnis von Bundespräsident und parlamentarischer Regierungsmehrheit der Großen Koalition besichtigt werden - und zwar in doppelter Hinsicht: zunächst mit Blick auf die Gesetzesqualität und die damit verbundene Legitimation des Gesetzgebers auf der Output-Ebene. Signifikant hierfür ist eine bemerkenswerte Kompensation der fehlenden eigenen Legitimation des Bundespräsidenten über eine Art präsidiales „Qualitätsmanagement“ für die legislativen Erzeugnisse der als parteiund machtpolitisch verdächtigten Mehrheiten im Parlament. So wird in den Medien eine offensive Rolle des Bundespräsidenten durchaus gern gesehen, ja sogar die Volkswahl des Präsidenten gefordert, damit er mit der Autorität der konkurrierenden Legitimation des volksgewählten Parlaments agieren könne ${ }^{40}$. Da dies aber kaum mehrheitsfähig ist, wird aus der gebotenen Neutralität des Amtes und der behaupteten ideologischen Inkongruenz beziehungsweise Indifferenz zu anderen Vetospielern ${ }^{41}$ eine apolitische Legitimitätsreserve abgeleitet, mit der sich die der Logik eines parlamentarischen Systems eigentlich zuwiderlaufende Aufwertung des Präsidenten durch seine Vetomacht nunmehr nicht mehr inputorientiert (wie in der Weimarer Republik), sondern output-orientiert begründen lässt: Der Präsident verhindert mit der im Prüfungsrecht verankerten „Vorbeugefunktion “ 42 bei der Ausfertigung von Gesetzen offenkundige Mängel des Gesetzgebungsverfahrens. ${ }^{43}$ Die damit einhergehende subkutane Botschaft lautet dann: „Exekutive und Legislative können es nicht. Köhler wirkt nicht als oberster Pädagoge, der Fehler tadelt, sondern er bläst über die

38 Federalist Nr. 48 (Madison), in: Clinton Rossiter (Hrsg.), The Federalist Papers. Hamilton, Madison, Jay, New York 1961, S. 313.

39 Michael Stoiber, Gewaltenteilung, Machtteilung und das Vetospieler-Konzept, a.a.O., S. 31.

40 Vgl. Punkt 5 in dem unsäglichen Zwölf-Punkte-Plan von Christoph Schwennicke, So retten wir Deutschland, in: http://www.sueddeutsche.de/deutschland/artikel/976/87889/print.html, sowie die angemessene Kommentierung von Frank Decker, Sehnsucht nach dem starken Mann. Wenn sich Journalisten als Verfassungsreformer versuchen, wird es meistens platt. Eine Widerrede, in: Berliner Republik, 2/2007.

41 Hierzu George Tsebelis, Decision Making in Political Systems. Veto Players in Presidentialism, Parliamentarism, Multicameralism and Multipartyism, in: British Journal of Political Science, 25. Jg. (1995), S. $289-326$.

42 Хиеши Gu, Die „Vorbeugefunktion“ des Bundespräsidenten, in: ZParl, 30. Jg. (1999), H. 3, S. $761-771$.

43 Zumal in einer Großen Koalition, mit einer Opposition, die weniger als ein Drittel der Abgeordneten stellt und keine Normenkontrollen beim BVerfG anzustrengen vermag, was wiederum „eine Aufforderung zur Nachlässigkeit an die Große Koalition“ sei, wie überhaupt die Qualität der Gesetzgebungsarbeit nachgelassen habe (vgl. Sebastian Fischer, a.a.O., S. 2, sowie auch die Kritik an der Gesetzeskontrolle durch das Bundesjustizministerium bei Nomos, Der Präsident als Staatsnotar, in: Die Zeit vom 14. Dezember 2006, S. 10, wo unter anderem auch insinuiert wird, neben einer schlampigen Kontrollarbeit fehle es dort womöglich auch ,an Autorität, um sich gegenüber politischen Zumutungen aus der Exekutive oder Legislative durchzusetzen"). 
Malaisen des Parlamentarismus noch einen Hauch von Vergeblichkeit. “44 Damit wäre in das parlamentarische System eine präsidiale Vetomacht installiert, die ihre Legitimation eben nicht mehr konkurrierend zur Legislative über die Input-Seite, sondern über die Output-Seite bezieht, dabei aber weiterhin die politische Prämie für eine aus dem Amt resultierende Neutralitätsvermutung beansprucht ${ }^{45}$ und zudem über eine spezifische Interpretation der Gewaltenteilung im Verhältnis von Legislative und Bundespräsident als Garant des demokratischen Prozesses und der Stabilität und Funktionalität des parlamentarischen Systems figuriert ${ }^{46}$. Dies ist die zweite Dimension des angesprochenen Verlustes der bona fideVermutung gegenüber dem Parlament. Sie ist durch eine eigentümliche anti-majoritäre Verwischung des Gewaltenteilungsdenkens geprägt, der sogleich noch ausführlicher nachzugehen ist.

Gleichwohl sei unter diesen Vorzeichen schon hier behauptet, dass die Vetomacht des Bundespräsidenten bei der Ausfertigung von Gesetzen nicht nur ein klassifikatorisches oder typologisches Problem ist und eben nicht etwas abschätzig "gewissen Verrichtungen “ zugeschlagen werden kann, um sodann zu konstatieren, diese könnten „die Stellung des Bundespräsidenten weder bestimmen noch fundieren, vielmehr wird umgekehrt die prinzipielle theoretische Fixierung der Rolle des Staatsoberhauptes in der parlamentarischen Demokratie vielleicht manche Zweifel erhellen, die durch unklare positivrechtliche Bestimmungen entstanden sind“ 47 . Das Problem ist vielmehr, dass es eine solche "prinzipielle theoretische Fixierung " der demokratischen Gewaltenteilung im parlamentarischen Bundesstaat genauso wenig gibt wie eine verfassungsrechtlich eindeutige Bestimmung des Umfangs des Prüfungsrechts aus Art. $82 \mathrm{GG}^{48}$. Weil dies so ist, werden Bestimmungsversuche seitens der Politikwissenschaft fast ausschließlich über „relational properties of democratic regime types “ 49 unternommen. Darunter sind „descriptions about the actual power situation“ beziehungsweise ein ,set of power relations that may be seen to occur within any given institutional framework" zu verstehen. ${ }^{50}$ Damit aber wird jeder Bestimmungsversuch kontingent und hängt von der jeweils situativ und subjektiv vorzunehmenden Einschätzung einer gewaltenteilig relevanten Akteurskonstellation ab, bei der der institutionelle Kontext, also die durch die Verfassung vorgegebenen Handlungskorridore und das dieses Design beherrschende Gewaltenteilungskonstrukt unbestimmt sind.

Diese Unbestimmtheit gilt übrigens auch für die Staatsrechtslehre, wenn etwa Andreas von Arnauld konstatiert, dass dem Gewaltenteilungssystem des Grundgesetzes - im Sinne verfassungsrechtlich fundierter Komplexe von Macht und Gegenmacht - eine eigenständige normative Bedeutung nur in begrenztem Maße zukomme, weswegen die verfassungsrechtliche Überprüfung konkreter Machtverschiebungen an den gewaltenteiligen Subsyste-

44 Thomas E. Schmidt, Das neue Jahr, in: Die Zeit vom 28. Dezember 2006, S. 3.

45 Dies ist letztlich die Konsequenz der Argumentation bei Robert Leicht, Beifall für die Person, nicht für das Programm, in: Die Zeit vom 1. März 2007, S. 6.

46 So explizit etwa Xuewu Gu, a.a.O, S. 771.

47 Otto Kimminich, Das Staatsoberhaupt in der parlamentarischen Demokratie, in: VVDStRL, 25 (1967), S. 2 - 94, S. 80.

48 Brun-Otto Bryde, Art. 82, in: Philip Kunig (Hrsg.), Grundgesetz-Kommentar, Bd. 3, 4./5., neubearbeitete Auflage, München 2003, S. 289, Rz. 21.

49 Robert Elgie, a.a.O. (2004), S. 316.

50 Ders., The Classification of democratic Regime Types: Conceptual ambiguity and contestable assumptions, in: European Journal of Political Research, 33. Jg. (1998), S. 219 - 238, S. 224. 
men anzusetzen habe. ${ }^{51}$ Die nicht fixierbare normative Bedeutung der grundgesetzlichen Gewaltenteilung ist nämlich das Resultat ihrer nicht eindeutigen institutionellen (und materiellen) Zuordnung. Die (rechtsdogmatisch wie typologisch) salvatorische Überprüfung anhand von Machtverschiebungen in Subsystemen - etwa im Verhältnis von Bundespräsident und Legislative - ist aber wenig hilfreich, wenn diese der gleichen Logik folgen und ebenfalls nicht festgelegt, sondern wie in Art. 82 GG rein „relational“ und deshalb kontingent angelegt sind. Somit kommt es letztlich auf die Interaktion von Akteuren im Gesetzgebungsprozess an, die changieren kann. Daraus folgt wiederum: Wenn als Gegenstand der vergleichenden Parlamentarismusforschung richtigerweise „nicht nur das Organ Parlament selbst (einschließlich seiner Akteure), sondern die Interaktion dieses Organs mit seiner politischen Umwelt" 52 gelten darf, dann gibt die in der Bundesrepublik Deutschland zuletzt aufscheinende Präsidentialisierung im institutionellen Rahmen eines parlamentarischen Systems Anlass zum Nachdenken, da sich damit zum einen das institutionelle Netz von Vetospielern und Vetopunkten noch verdichten würde und zum anderen ein eigentlich „ruhender“ institutioneller Vetospieler aus seiner Reservefunktion geholt und zum „regierenden Repräsentanten" 53 mutieren würde, der nahezu zwangsläufig in parteipolitische Auseinandersetzungen und in die verfassungspolitische Diskussion geriete ${ }^{54}$.

Das ohnehin umstrittene allgemeine Verständnis davon, was unter „parlamentarisches System " fällt ${ }^{55}$, dürfte damit nicht vereinfacht werden und die von Dolf Sternberger schon früh artikulierte Vermutung, dass es sich bei der parlamentarischen Regierungsform in der Bundesrepublik streng genommen eigentlich um eine „entartete Regierungsform “56 handele, neue Nahrung erhalten. Oder noch etwas zugespitzter formuliert: Einen so „parlamentarischen Parlamentarismus " 57 haben wir unter den Auspizien eines Bundespräsidenten als „Außerparlamentarische Opposition“ sowie einer dies begünstigenden Mischverfassung vielleicht gar nicht.

\section{Prüfungsrecht und Ausfertigungsverweigerung: Bundespräsident und Parlament in der konsensdemokratischen Mischverfassung der Bundesrepublik}

In Analysen des parlamentarischen Systems der Bundesrepublik Deutschland hat der Bundespräsident bislang gar keine oder eine nur marginale Rolle gespielt ${ }^{58}$ - er gehört offen-

51 Andreas von Arnauld, a.a.O., S. 698.

52 Susanne S. Schüttemeyer, Vergleichende Parlamentarismusforschung, in: Dirk Berg-Schlosser / Ferdinand Müller-Rommel (Hrsg.), Vergleichende Politikwissenschaft, 4., überarbeitete und erweiterte Auflage, Opladen 2003, S. 207 - 227, S. 210.

53 Sebastian Fischer, a.a.O., S. 3.

54 Vollkommen zutreffend in dieser Hinsicht Brun-Otto Bryde, a.a.O., Rz. 21 mit Hinweis auf die Misslichkeit der inkonsequenten herrschenden Lehre.

55 Stefan Marschall, a.a.O., S. 67.

56 Dolf Sternberger, Gewaltenteilung und parlamentarische Regierung in der Bundesrepublik Deutschland (1960), in: Theo Stammen (Hrsg.), Strukturwandel der modernen Regierung, Darmstadt 1967, S. 186 - 209, S. 199.

57 Peter Lösche, Der Bundestag - kein ,trauriges', kein ,ohnmächtiges‘ Parlament, in: ZParl, 31. Jg. (2000), H. 4, S. 926 - 936, S. 926.

58 Patrick Horst, Präsident der Bundesrepublik Deutschland: Der Rang des Amtes und die Folge der Amtsinhaber, in: ZParl, 26 Jg. (1995), H. 4, S. 586 - 594, S. 586. 
kundig nicht zu den „Schlüsselinstitutionen des Regierungsbetriebs“59 und wurde auch in älteren Darstellungen als „keine wichtige Figur im politischen Kräftefeld der Bundesrepublik"60 beschrieben. Gerade „in Zeiten klarer parlamentarischer Mehrheiten“" sei er auf eine staatsnotarielle oder Beurkundungsfunktion beschränkt ${ }^{61}$, weshalb er in neueren Arbeiten zum Parlamentarismus beziehungsweise zum Bundestag als Gesetzgeber als intervenierende Variable und / oder Vetospieler im Gesetzgebungsverfahren nicht vorkommt ${ }^{62}$. Dies deckt sich mit der Diagnose von Juan Linz, dass die Politikwissenschaft bei der Analyse parlamentarischer Systeme die Staatsoberhäupter ausblende ${ }^{63}$, lässt sich aber damit erklären, dass die empirischen Beispiele für ein explizites „Vetospielen“ des Bundespräsidenten bislang nicht zahlreich gewesen sind ${ }^{64}$. Zudem hat das Grundgesetz dem Bundespräsidenten im Vergleich zur Weimarer Reichsverfassung eine zurückgenommene Rolle zugewiesen, so dass die Grundlagen für eine „allgemeine Theorie der Entfesselung präsidentieller Macht im deutschen Regierungssystem" 65 - bislang jedenfalls - zu fehlen schienen und, so die gängige Vermutung, ein starkes Parlament sich ohnehin „eine eigene Regierungspolitik des Staatsoberhauptes nicht gefallen lassen“" würde. ${ }^{66}$ Gleichwohl bleibt das Insistieren berechtigt, dass es sich beim Staatsoberhaupt in parlamentarischen Demokratien um mehr als ein monarchisches Relikt handeln müsse - obwohl auch der Bundespräsident und seine Kompetenzen (vor allem Gegenzeichnung) regelmäßig so kategorisiert werden ${ }^{67}$-, denn „if presidents in pure parliamentary republics were irrelevant, it would not make sense for politicians to put so much effort into electing their preferred candidate to the office" 68 .

59 Jürgen Hartmann, Das politische System der Bundesrepublik Deutschland im Kontext. Eine Einführung, Wiesbaden 2004, S. $143 \mathrm{ff}$.

60 Kurt Sontheimer, Grundzüge des politischen Systems der Bundesrepublik Deutschland, überarbeitete Neuausgabe, 9. Auflage, München 1984, S. 246.

61 Wolfgang Rudzio, a.a.O., S. 295.

62 Vgl. Heinrich Oberreuter / Uwe Kranenpohl / Martin Sebaldt (Hrsg.), Der deutsche Bundestag im Wandel. Ergebnisse neuerer Parlamentarismusforschung, Wiesbaden 2001; Klaus von Beyme, Der Gesetzgeber. Der Bundestag als Entscheidungszentrum, Wiesbaden 1997.

63 Juan Linz, Presidential or Parliamentary Democracy: Does it make a Difference?, in: Juan Linz / Arturo Valenzuela (Hrsg.), a.a.O., S. 3 - 87, S. 46.

64 Vgl. die Übersichten bei Johannes Rau, Vom Gesetzesprüfungsrecht des Bundespräsidenten, in: Peter Häberle / Martin Morlok / Vassilios Skouris (Hrsg.), Festschrift für Dimitris Th. Tsatsos zum 70. Geburtstag, Baden-Baden 2003, S. 562 - 576, S. 566 ff., sowie bei Volker Epping, Das Ausfertigungsverweigerungsrecht im Selbstverständnis der Bundespräsidenten, in: JZ, 23 (1991), S. 1106 ff. Brun-Otto Bryde, a.a.O., Rz. 21 betont allerdings, dass dies eigentlich nur ein Zufall sei, weil die bisherigen Bundespräsidenten alle einer Regierungspartei angehört hätten, weswegen sie sich der parlamentarischen Mehrheit gegenüber Zurückhaltung auferlegt hätten, während die Opposition in sie wiederum keine übertriebenen Erwartungen gesetzt hätte.

65 Ludger Helms, Regierungsorganisation und politische Führung in Deutschland, Wiesbaden 2005, S. 169.

66 Ernst Friesenhahn, Parlament und Regierung im modernen Staat, in: VVDStRl, 16 (1958), S. 9 - 73, abgedruckt in: Theo Stammen (Hrsg.), a.a.O., S. 109 - 185, S. 148.

67 Vgl. Klaus von Beyme, a.a.O. (1999), S. 332, sowie Brun-Otto Bryde, Art. 82, a.a.O, S. 289, Rz. 21.

68 Juan Linz, Presidential or Parliamentary Democracy: Does it make a Difference?, in: Juan Linz / Arturo Valenzuela (Hrsg.), a.a.O., S. 46. Dies gilt auch für die Bundesrepublik Deutschland, wo die Wahl des Bundespräsidenten „stark von parteipolitischen Überlegungen und Taktiken bestimmt ist", die sich auf die jeweils bevorstehenden Bundestagswahlen und mögliche Koalitionsbildungen fokussieren, so Werner Billing, Der Kampf um die Besetzung des höchsten Staatsamtes: Auswahl und Wahl des Bundespräsidenten 1994, in: ZParl, 26. Jg. (1995), H. 4, S. 595 - 620, S. 596. 
Und in der Tat: Das Grundgesetz zeigt bei näherer Betrachtung, „daß der Bundespräsident nicht das schlechthin apolitische Verfassungsorgan ist, als das er mitunter dargestellt wird"69, wobei seine Stellung insbesondere dann gestärkt zu werden scheint, „wenn in der Verfassungspraxis Schwierigkeiten entstehen, wenn es zum Konflikt zwischen Parlament und Regierung kommt, also die Grundvoraussetzungen des parlamentarischen Systems, das Miteinander von Regierung und Parlamentsmehrheit, nicht mehr gegeben ist" "70. Die metaphorisch reizvoll mit der Funktion des Staatsnotars spielende Formulierung, der Bundespräsident habe bei der Verweigerung der Ausfertigung zweier Gesetze der Großen Koalition „den unbenutzten Füller "71 gezeigt, weist auf seine eigentümliche Reservefunktion hin ${ }^{72}$, die allerdings hinsichtlich ihrer grundgesetzkonformen Tragweite gerade mit Blick auf das materielle Prüfungsrecht nach Art. 82 GG in der Staatsrechtslehre seit jeher umstritten ist und dort mit einem seit längerem "geschlossenen Argumentationshaushalt" ${ }^{\text {"73 }}$ diskutiert wird. Die so hingebungsvoll geführte Diskussion um die Existenz eines materiellen Prüfungsrechts beziehungsweise dessen Reichweite geht allerdings an der eigentlich relevanten Machtfrage, die wiederum für das dahinter stehende Problem der Gewaltenteilung ausschlaggebend ist und letztlich auch hinter den gängigen Typologisierungen der Komparatistik steht, schlichtweg vorbei. Die Existenz eines materiellen Prüfungsrechts ist nämlich erst bedeutsam, wenn daraus ein Ausfertigungsverweigerungsrecht abgeleitet wird ${ }^{74}$; erst in diesem Fall kommt es zu einer faktischen Vetofunktion des Bundespräsidenten und gegebenenfalls zu einer Verschiebung der Koordinaten des parlamentarischen Systems und damit $\mathrm{zu}$ „konkreten Machtverschiebungen in einem gewaltenteiligen Subsystem “75. Die Verwerfungskompetenz des Bundespräsidenten ist im Übrigen weitgehend unstreitig gegeben, jedenfalls soweit der Bundespräsident zu der Auffassung gelangt, ein Gesetz sei nicht nach den Vorschriften des Grundgesetzes zustande gekommen. Somit erstreckt sich dieses formelle Prüfungsrecht üblicherweise auch auf die Frage der Einhaltung der Kompetenzvorschriften. ${ }^{76}$ Wesentlich strittiger ist aber die Konsequenz aus der erfolgten Prüfung: Muss der Bundespräsident, wenn er zu einem negativen Urteil kommt, die Ausfertigung verweigern, weil ihm etwa nicht zuzumuten ist, ein verfassungswidriges Gesetz auszufertigen und weil alle Verfassungsorgane gleichermaßen zum Schutz der Verfassung berufen sind? Oder kann beziehungsweise soll er trotz seiner eigenen negativen Einschätzung der Beurteilungsprärogative des parlamentarischen Gesetzgebers den Vorrang lassen, ausfertigen und die

69 Christoph Degenhart, a.a.O., S. 191, Rz. 456.

70 Joachim Jens Hesse / Thomas Ellwein, Das Regierungssystem der Bundesrepublik Deutschland, 7., völlig neubearbeitete und erweiterte Auflage, Bd. 1, Opladen 1992, S. 297.

71 Thomas E. Schmidt, a.a.O., S. 3.

72 Explizit gegen eine bloße Reservefunktion Xuеwu Gu, a.a.O., S. $763 \mathrm{ff}$.

73 Philip Kunig, Der Bundespräsident, in: Jura 1994, S. 217 - 222, S. 220.

74 Vollkommen zutreffend in dieser Hinsicht Volker Epping, a.a.O., S. $1102-1110$, besonders S. $1106 \mathrm{ff}$.

75 Andreas von Arnauld, a.a.O., S. 698.

76 Hans D. Jarass / Bodo Pieroth, Grundgesetz für die Bundesrepublik Deutschland. Kommentar, 7. Auflage, München 2004, Art. 82, Rz. 3. Eine Verletzung der Kompetenzvorschriften des Grundgesetzes stand sowohl beim LuftSiG als auch beim Gesetz zur Neuregelung des Rechts der Verbraucherinformation und beim Gesetz zur Neuregelung der Flugsicherung in Rede - in allen drei Fällen hat der Bundespräsident hierauf Bezug genommen, in den beiden letztgenannten Fällen, um seine Ausfertigungsverweigerung zu begründen, im erstgenannten Fall, um zumindest schwerwiegende Bedenken gegen die Neuregelung geltend zu machen. 
endgültige Beurteilung dem Bundesverfassungsgericht überlassen? Man kann diese Frage zunächst dahingehend differenzierend beantworten, dass die Evidenz einer materiellen Verfassungswidrigkeit sich jedenfalls noch nicht dann ergibt, wenn es sich nur um einen „Dissens über die Verfassungskonformität im Bereich des Spektrums unterschiedlicher, aber jeweils vertretbarer Auffassungen "77 handelt - in einem solchen Falle sei der Bundespräsident zur Ausfertigungsverweigerung nicht berechtigt. Erst bei evidenter Verfassungswidrigkeit könne somit ein Verweigerungsrecht des Bundespräsidenten entstehen. Auf diese Evidenz rekurrieren die Begründungen des Bundespräsidenten für die Verweigerung der Ausfertigung ganz explizit ${ }^{78}$, und so wurden sie auch rezipiert:

„Wenn man näher hinschaut, ging Köhler in den drei genannten Fällen genau abgestuft vor: Beim sogenannten Flugzeugabschuss-Gesetz handelte es sich um eine verfassungspolitische Abwägungs- und Interpretationsfrage, also unterzeichnete Köhler unter Anführung schwerer Bedenken, überließ aber das letzte Wort (und Veto) den Bundesverfassungsrichtern. Angesichts der politischen Implikationen war dies die schonendste Form der Intervention. Bei der Privatisierung der Luftraumüberwachung stand schon der klare Wortlaut des Grundgesetzes im Wege. Köhler konnte gar nicht anders, als seine Unterschrift zu verweigern, wobei er zusätzlich klar machte (wiederum ein Ausdruck politischer Zurückhaltung!), dass sein Veto sich nicht gegen die politische Absicht des Gesetzgebers richtet; man könne ja das Grundgesetz gegebenenfalls ändern. Beim Verbraucherschutzgesetz war es nicht der Präsident, der über den Zaun gefressen hatte, sondern der Gesetzgeber selbst: Es fehlte ihm nämlich schlicht die Zuständigkeit."79

Interessanter als die Frage nach der Existenz eines materiellen Prüfungsrechts und dessen Reichweite bleibt indessen, wie die Verwerfungskompetenz des Bundespräsidenten bei der Gesetzesausfertigung gewaltenteilungstheoretisch in den parlamentarischen Bundesstaat eingepasst werden kann. Hierzu hat sich die deutsche Politikwissenschaft bislang weitgehend bedeckt gehalten; umso zahlreicher sind die Beiträge hierzu aus der Staatsrechtslehre. Sie zeichnen sich zum einen dadurch aus, dass sie die im Grundgesetz angelegte Gewaltenteilung durchaus zutreffend in der Tradition der Mischverfassung lesen, zum anderen aber diese Gewaltenteilung als „nicht im Sinn einer durchgehend scharfen Trennung verwirklicht ${ }^{\text {“ } 80}$, sondern vielmehr mittels eines „aufgabenteiligen Kooperationsmodells“ 81 durchbrochen sehen, wobei wiederum der Bundespräsident eine besonders oszillierende Position einnehme, die gerade gegenüber der parlamentarischen Regierung deutlich werde. Somit müsse der Versuch einer eindeutigen Zuordnung des Bundespräsidenten in einem System der (ohnehin nicht strikt durchgeführten) Gewaltenteilung fehlschlagen; „auch das Amt des Bundespräsidenten lässt sich nicht - nicht einmal negativ - einer der klassischen Gewalten zurechnen. Es gehört, auch als Kontrollorgan, weder zur Exekutive noch zur Legislative oder gar zur Judikative, die gerade in Bezug auf die Wahrung der Verfassung im Bundesverfassungsgericht einen besonderen Auftrag erhalten hat. Die Auffassung, daß sich das Wirken des Bundespräsidenten gegenüber der parlamentarischen Regierung ,nur verhindernd, nicht unternehmend' zu äußern vermöge, ist zu eng. Sie bezieht sich nur auf einige Funktionen des Bundespräsidenten, nicht auf deren Gesamtheit. " 82

79 Nomos, a.a.O., S. 10.

80 Christoph Degenhart, a.a.O., S. 195, Rz. 465.

81 Andreas von Arnauld, a.a.O., S. 680.

82 Jürgen Jekewitz, a.a.O., vor Art. 54, S. 479, Rz. 18. 


\section{Der Bundespräsident als nicht immer neutralisierbarer Vetospieler: die anti-majoritäre Hybridisierung des parlamentarischen Regierungssystems}

Nach den bisherigen Ausführungen wird deutlich, dass der Präsident sowohl neben als auch mitten in der gewaltenteiligen Ausübung relevanter staatlicher Funktionen steht (und stehen soll): „Er nimmt an der Ausübung der Staatsgewalt in funktionaler und gewaltenteiliger Verschränkung mit anderen Organen teil. Diese Verschränkung ist Kennzeichen moderner Verfassungen, die vom demokratischen Prozess beherrscht sind und den Anforderungen der heutigen Gesellschaft gerecht werden. Diese Verschränkung ist Ausdruck der Idee der gemischten Verfassung, in der Organe verschiedenen Zuschnitts die Ausübung der Staatsgewalt zugleich fördern und kontrollieren. "83 Dies manifestiert sich besonders im Gesetzgebungsprozess beziehungsweise der Verwerfungskompetenz des Bundespräsidenten nach Art. 82 GG. Zwar wirkt er „nicht am Zustandekommen eines Gesetzes mit, ist aber im Gesetzgebungsverfahren beteiligt: Er erwirkt mit der Ausfertigung eines Gesetzes den letzten vor der Verkündung und dem Inkrafttreten des Gesetzes liegenden Akt des Gesetzgebungsverfahrens. Diese Teilhabe am Legislativprozess dient nicht lediglich notarieller Fixierung einer von anderen Organen getroffenen Entscheidung, sondern ist unter dem Aspekt der Prüfungskompetenz des Bundespräsidenten ein konstituierender Bestandteil des demokratischen Prozesses der Rechtsetzung “84. Diese Einschätzung ist äußerst interessant, da der Bundespräsident hier ganz selbstverständlich als konstitutiver Bestandteil des demokratischen Prozesses figuriert, dem er doch gerade enthoben sein soll, besonders wenn es sich um dessen parteipolitische Dimension handelt. Offenkundig wird der demokratische Prozess hier durch die bereits angesprochene Madison-Brille betrachtet ${ }^{85}$, die einem zu wenig institutionell gehegten demokratischen Prozess und damit einem potentiellen Parlamentsabsolutismus als auch den dahinter stehenden Mehrheiten und ihren Beschlüssen zutiefst misstraut, getreu der Diagnose "that measures are too often decided, not according to the rules of justice and the rights of the minor party, but by the superior force of an interested and overbearing majority "86. Der demokratische Prozess muss also prozedural und materiell einem „laundering“ unterzogen werden - klassisches Attribut für ein Denken in institutionellen Kategorien ${ }^{87}$. Unter den Parametern eines parlamentarischen Regierungssystems kann aber eine solche „Weißwäsche“ in Gestalt einer präsidialen Prüfungs- und Vetokompetenz bei fehlender Legitimation durch das Volk nur positiv gewendet werden, wenn die Ausfertigung von Gesetzen durch den Bundespräsidenten als Veranschaulichung des Abschlusses der politischen Auseinandersetzung dargestellt und ihnen somit attestiert wird, sie würden symbolisch die allgemeine Verbindlichkeit der Beschlüsse zum Ausdruck bringen: „Das so ausgefertigte und im Gesetzesblatt erscheinende Gesetz ist ein Parlamentsgesetz, nicht länger ein Beschluß der Parlamentsmehrheit. "88 Das betrifft den

83 Martin Nettesheim, a.a.O., S. 1054 f., Rz. 41.

84 Ebenda, S. 1054 f., Rz. 42.

85 Prononciert in diese Richtung vor allem Matthew S. Shugart, a.a.O. (2006); ders, a.a.O. (2005), S. $328 \mathrm{ff}$.

86 Federalist No. 10 (Madison), in: Clinton Rossiter (Hrsg.), a.a.O., S. 77.

87 Hierzu Roland Lhotta, Der antiliberale Kern des neuen Institutionalismus in der Politikwissenschaft: Überlegungen zum aktuellen Erscheinungsbild der Disziplin, in: ZPol, 16. Jg. (2006), S. 7 - 28.

88 Klaus Schlaich, a.a.O., S. 552, Rz. 25. 
positiven (Regel-)Fall der Ausfertigung; was aber ist mit dem negativen Fall der Nichtausfertigung? Dieser Logik zufolge bliebe ein nicht ausgefertigtes Gesetz nämlich ein bloßer Mehrheitsbeschluss, was offenkundig negativ konnotiert ist und auf ein Legitimations- und Kontrolldefizit verweist, hinter dem sich die „Tyrannei der Mehrheit“ verbirgt, die wiederum in der Legislative am ehesten drohe ${ }^{89}$. Diese wäre ein Verstoß gegen das in der nicht trennscharf angelegten Mischverfassung des Grundgesetzes institutionalisierte „Prinzip der kooperativen Gewaltenteilung "90 und (zumindest potentiell) das Resultat eines möglicherweise „verfassungswidrigen Zusammenwirkens der anderen Verfassungsorgane“, weswegen man diese eben „nicht ganz sich selbst überlassen "91 sollte. Somit ist es auch ganz folgerichtig, dass das Prüfungsrecht des Präsidenten als zusätzliche Form der Mitkontrolle funktioniert, welche der Erhaltung des demokratischen Systems diene - eines Systems, das offensichtlich einen „surplus“ gegenüber der Demokratie als Verfahren zur Herstellung von (parlamentarischen) Entscheidungen aufweist, weil hier der Rechtsstaat mit dem Bundespräsidenten als seinem präsumtiven Agenten gegen einen möglichen Parlamentsabsolutismus in Stellung gebracht wird ${ }^{22}$.

Damit aber wird der für das parlamentarische System der Bundesrepublik so selbstverständlich tradierte „Lehrsatz“, der Bundespräsident trete in Zeiten stabiler parlamentarischer Mehrheiten in den Hintergrund und sei nur bei „Krisen“ gefordert, fragwürdig und erscheint gerade durch die jüngste Staatspraxis unter Horst Köhler in zweifelhaftem Licht. Vielmehr wird gerade die Konstellation einer Großen Koalition mit ihrer „super-majority“ und der damit verbundenen Gefahr einer Tyrannei der Mehrheit zum eigentlichen Krisenfall parlamentarischer Demokratie, in dem der Bundespräsident aus Gründen der gewollt so wenig trennscharfen Gewaltenteilung und des Schutzes der Verfassung besonders wachsam zu sein habe. Diese Sichtweisen entstammen einem wohlbekannten älteren Diskussionskontext, in dem das Staatsoberhaupt als dem Parteienwettbewerb enthobener „pouvoir neutre“ agiert $^{93}$; sie finden sich explizit oder implizit in eigentlich allen staatsrechtlichen Beiträgen zu dieser Thematik und besonders pointiert auch in Ausführungen Theodor Eschenburgs:

„Der Umstand, daß der Bundespräsident die Gesetze wirksam auf ihre Verfassungsmäßigkeit prüfen kann, gibt den gesetzgebenden Organen Veranlassung, bei der Verabschiedung die erforderliche Sorgfalt walten zu lassen. Bundesregierung, Bundesrat und Bundestag sind parteipolitisch zusammengesetzte Gremien, sie könnten geneigt sein, den Zweck höher zu bewerten als die Verfassungsmäßigkeit eines Gesetzes. Hier ein über den Parteien stehendes Gegengewicht zu bilden, ist Aufgabe des Bundespräsidenten. Er wirkt hier als ,Hüter der Verfassung ' (...) Daß ein so kompliziertes Verfahren zur Prüfung der Gesetze und ihrer etwaigen Anfechtung erforderlich ist, könnte verwunderlich erscheinen. Und doch hat es seinen guten Grund: die um die Willensbildung miteinander Ringenden sind geneigt, unter dem Gesichtspunkt der angestrebten Zwecke die Schranken der Verfassung zu gering oder zu hoch zu veranschlagen.

89 So mit Verweis auf Federalist Papers Nr. 52 - 66: Manfred G. Schmidt, Demokratietheorien, a.a.O., S. 119; vgl. auch Michael Stoiber, a.a.O., S. 28 f.

90 Johannes Rau, a.a.O., S. 564.

91 Klaus Schlaich, a.a.O., S. 559 f., Rz. 41.

92 Insofern verkennen Norbert K. Riedel / Axel Schmidt, a.a.O., S. 374, die Affinität der gewaltenteilungstheoretisch prekären Prüfungskompetenz des Bundespräsidenten zur Lehre von den checks and balances, wenn sie diese mit dem rein formalistischen Verweis auf die ungleich größeren Kompetenzen des amerikanischen Präsidenten ablehnen.

93 Umfassend dazu Otto Kimminich, a.a.O. 
Diejenigen, die einen bestimmten Zweck zu erreichen trachten, wollen es sich so einfach wie möglich machen, diejenigen, die die Erreichung dieses Zweckes verhindern möchten, wollen es den anderen so schwer wie möglich machen. Durch diesen Auseinandersetzungsprozeß entsteht leicht eine Verformung der Institutionen und dadurch Rechtsunsicherheit. Denn nur der Zweck ist gerechtfertigt, der mit rechtmäßigen Mitteln erreicht wird. Was aber rechtmäßig ist, kann im Streit der Zwecke übersehen werden. “94

Es gibt hiernach Akteurskonstellationen im parlamentarischen Bundesstaat, in denen die „rules of the game“ der Verfassung unterlaufen oder auch überdehnt werden, weil diese ansonsten der Zweckerreichung politisch potenter Akteure - wie sie sich etwa in starken parlamentarischen Mehrheiten manifestieren können - entgegenstünden. Hier liegt dann die tiefere anti-majoritäre und rechtsstaatliche Ratio des Prüfungsrechts des Bundespräsidenten: „Gerade die Neigung von politischen Fuhrleuten, sich etwas auf ihre Mehrheiten einzubilden (und auf ihre übergroßen Mehrheiten noch etwas mehr), ist aber das entscheidende verfassungspolitische Motiv für das Prüfungsrecht des Bundespräsidenten “95, weswegen man auch flugs zu der Folgerung gelangt, die Staatspraxis belege, „dass ein Bedarf für ein materielles Ausfertigungsverweigerungsrecht des Bundespräsidenten als zusätzliche Sicherung zur Beachtung des Grundgesetzes besteht" $" 96$. Insofern sei eine Proliferation von Hütern der Verfassung sogar Sinn und Zweck einer nicht trennscharfen Mischverfassung und stelle auch keine Konkurrenz zum Bundesverfassungsgericht dar' ${ }^{97}$, denn der Bundespräsident verhindere im Falle der Nichtausfertigung eines Gesetzes dessen Inkrafttreten vor Abschluss des Gesetzgebungsverfahrens und realisiere damit den sofortigen Schutz des Vorrangs der Verfassung, der sonst nur im Wege einer einstweiligen Anordnung des Bundesverfassungsgerichts erreichbar wäre. ${ }^{98}$

Damit ist der Bundespräsident mit seinem Prüfungs- und Ausfertigungsverweigerungsrecht offenkundiger Bestandteil jenes tief gestaffelten Systems von checks and balances, dem sich parlamentarische Mehrheiten in der Bundesrepublik Deutschland gegenüber sehen. Als nachträglicher und fallabhängiger Vetospieler trägt er zur Intensivierung der Machtaufteilung durch Proliferation der Vetospieler sowie zur konsensdemokratischen Verhinderung einer „single concentration of power" bei. Er bleibt aber auch ein Vetospieler, der - in der Terminologie von Tsebelis - nicht ohne weiteres absorbiert werden kann, zumal nicht über parteipolitische Konsonanz und auch nicht über eine augenscheinlich nur vorgegebene Reservefunktion. Die dem Bundespräsidenten zukommende Entscheidung der Ausfertigungsverweigerung beendet den Gesetzgebungsprozess eben nicht nur vorläufig, sondern endgültig - und zwar ohne eine konkurrierende Legitimation durch den Volkssouverän, dafür aber mittels einer output-zentrierten sowie anti-majoritären Legalität, die gegen Parlamentsmehrheiten in Stellung gebracht wird. Hierin keine im Widerspruch zum parlamentarischen System stehende Kompetenz zu sehen ${ }^{99}$, scheint dann doch etwas be-

94 Theodor Eschenburg, Staat und Gesellschaft in Deutschland, Stuttgart 1956, S. 647 f. (Hervorhebungen des Verfassers).

95 Robert Leicht, a.a.O. (2006), S. 12.

96 Volker Epping, a.a.O., S. 1109.

97 Vgl. Gerold Lehngut, Die Verweigerung der Ausfertigung von Gesetzen durch den Bundespräsidenten und das weitere Verfahren, in: DÖV 1992, S. 439 ff., S. 442; Volker Epping, a.a.O., S. 1106; Klaus Schlaich, a.a.O., S. 557 f., Rz. 38.

98 Klaus Schlaich, a.a.O., S. 557 f., Rz. 38.

99 Knut Ipsen / Volker Epping, Der Staatsnotar blockt - Ein Bericht über eine öffentlichrechtliche Examensklausur, in: JuS 1992, S. 305 - 310, S. 309. 
müht, genauso wie die recht benevolente Attestierung einer krisenvorbeugenden Funktion samt deren abschreckender Wirkung für die Parlamentarier ${ }^{100}$.

„Präsidentialisierung des Präsidentenamtes“ und „parlamentarischer Parlamentarismus“ sind eben nicht ohne weiteres zur Deckung zu bringen - auch wenn man mit Winfried Steffani argumentieren kann, dass ein Ausfertigungsverweigerungsrecht des Bundespräsidenten weder die Hauptkriterien noch die supplementären Elemente parlamentarischer Systeme ${ }^{101}$ tangiert. Oder aber man konstatiert mit Robert Elgie, dass der Vetospieler-Ansatz ohnehin jede Systemtypologie unterlaufe und damit letztlich überflüssig mache ${ }^{102}$ - mithin könnte auch ein Präsident mit Vetomacht als eine Variante von ,institutional effects of regime types“ analysiert werden, ohne sich dem Zwang zur Typologisierung zu unterwerfen. Parlamentarisches, präsidentielles und semi-präsidentielles System seien damit letztlich überflüssige Kategorien. Beide Ansätze sind nicht befriedigend, da sie ein letztlich evaluatives Problem hintergehen, indem sie es entweder hinwegdefinieren oder aber ignorieren ${ }^{103}$. Eine Vetokompetenz des demokratisch gegenüber dem Parlament nachrangig legitimierten Staatsoberhauptes bleibt aber in jedem Fall ein Stachel im Fleisch des parlamentarischen Systems. Insoweit ist Brun-Otto Bryde zuzustimmen, wenn er mit Blick auf Art. 82 GG konstatiert, hier habe das Grundgesetz „für den formellen Abschluss des Gesetzgebungsverfahrens Traditionen des konstitutionellen Staatsrechts übernommen, ohne sie auf ihre Adäquanz für die Verfassungsordnung des Grundgesetzes kritisch zu überprüfen. Entgegen mancher Kritik ist zwar daran festzuhalten, daß die Ausfertigung der Gesetze durch das Staatsoberhaupt sinnvoll ist, wenn am Gesetzgebungsverfahren mehrere Organe beteiligt sind. (...) Auch hätte der Verfassungsgeber die äußerst umstrittene Frage des materiellen Prüfungsrechts eindeutiger klären müssen. Wenn der Bundespräsident ein solches Recht haben soll, muß die Verfassung das aussprechen und entsprechende Verfahrensvorkehrungen treffen, z.B. eine Aussetzung der Ausfertigung und Anrufung des BVerfG vorsehen "104.

Die konsequent bis auf die Subsysteme des Gewaltenteilungssystems eingehaltene Mischverfassung des Grundgesetzes macht gerade im Fall der Ausfertigung von Gesetzen das Verhältnis der Akteure im Gesetzgebungsprozess - hier: Parlament und Präsident - zu einer relationalen und kontingenten Angelegenheit, in der die Normen der Verfassung keinen Halt geben und zum Situationsrecht werden. Solange es gelingt, den Bundespräsidenten - wie bislang weitgehend der Fall - über die parteipolitische Zugehörigkeit zur Regierungsmehrheit oder aber im Wege der selbstverordneten Zurückhaltung als Vetospieler zu neutralisieren, mag das Staatsoberhaupt „figurehead“ bleiben. Das muss aber nicht immer so sein, wie sich gerade zeigt - und spätestens in solchen Situationen erhält das parlamentarische System der Bundesrepublik Deutschland hybride Züge und der Firnis der bloßen Reservefunktion des Bundespräsidenten einige unschöne Risse.

100 Xиewu Gu, a.a.O., S. $764 \mathrm{f}$.

$101 \mathrm{Vgl}$. Winfried Steffani, Parlamentarische und präsidentielle Demokratie. Strukturelle Aspekte westlicher Demokratien, Opladen 1979, S. 39 ff., sowie ders., a.a.O. (1995), S. 630.

102 Robert Elgie, a.a.O. (2004), S. 327.

103 Was allerdings ein Wesenszug der Politikwissenschaft neuer Provenienz zu sein scheint - vgl. hierzu Wolfgang Seibel, Suchen wir immer an der richtigen Stelle? Einige Bemerkungen zur politikwissenschaftlichen Forschung nach dem Ende des Kalten Krieges, in: PVS, 44. Jg. (2003), S. 217 - 228, sowie Roland Lhotta, a.a.O., passim.

104 Brun-Otto Bryde, Art. 82, a.a.O., S. 289, Rz. 21. 